Годишњак Филозофског факултета у Новом Саду, Књига XLVI-3 (2021)

Annual Review of the Faculty of Philosophy, Novi Sad, Volume XLVI-3 (2021)

Milena Kasaposka-Chandlovska*

Université «St. Clément d'Ohrid »

Bitola

Maja Milevska-Kulevska

Université « Mère Teresa » Skopje
UDK : 811.133.1'243:371.3(497.17)

DOI: $10.19090 /$ gff.2021.3.139-153

originalni naučni rad

\title{
LA GRAMMAIRE DANS LES MANUELS DE FLE UTILISÉS DANS L'ENSEIGNEMENT PRIMAIRE EN MACÉDOINE
}

Ayant en vue qu'en Macédoine le nombre d'élèves choisissant le français en primaire ne cesse de diminuer les dernières années et sachant que ce manque d'intérêt a des implications directes sur l'apprentissage du français dans les niveaux supérieurs d'éducation, il est tout à fait légitime de se demander quelles en sont les raisons possibles. Interrogés pourquoi ils ne font pas le choix du français au niveau universitaire, la majorité de ceux l'ayant déjà appris en primaire avancent que le manque d'intérêt pour le français est dû à la complexité de sa grammaire. Le fait même qu'ils résument l'apprentissage du français à l'acquisition de sa grammaire peut indiquer que malgré les approches didactiques modernes recommandées depuis plus de vingt ans par le Cadre européen commun de référence (CECRL), il s'avère possible que la grammaire dans les manuels utilisés en classe de FLE ne soit pas au service des objectifs de communication mais qu'elle soit toujours représentée d'une manière " traditionnelle ». Dans ce sens, l'objectif de cette étude sera de faire une analyse des contenus grammaticaux présents dans deux manuels de FLE actuellement en usage dans l'enseignement primaire en Macédoine, à savoir Le Mag et Vite. En premier lieu, l'analyse nous permettra de voir quelle est la représentation de la grammaire dans ces deux manuels et de vérifier dans quelle mesure elle correspond aux recommandations du CECRL pour le développement des compétences communicatives. En deuxième lieu, nous allons analyser le type d'approche grammaticale dans les manuels ainsi que le type d'exercices proposés pour son acquisition.

Mots-clés : grammaire, manuels, français, primaire, Macédoine.

\section{INTRODUCTION}

Depuis plusieurs années déjà les enseignants de français en Macédoine témoignent d'un décroissement continu de l'intérêt pour l'apprentissage de la

\footnotetext{
*milena.kasaposka@uklo.edu.mk
} 
langue française. Cette situation a des implications directes sur le statut du français au niveau secondaire où l'élève est censé continuer l'apprentissage de la langue étrangère qu'il a apprise au primaire. Logiquement, cette réalité se reflète également au niveau universitaire.

Laissant de côté les autres raisons possibles pour la situation défavorable dans laquelle se trouvent les enseignants du français, il ne faudrait, à notre avis, négliger l'opinion des élèves que le français est une langue complexe et que sa grammaire est beaucoup plus difficile en comparaison avec la plupart des autres langues étrangères. Au fait, ces réflexions ne sont pas nouvelles et méritent d'être prises en considération lors de l'élaboration des curriculums pour le FLE en Macédoine.

La plupart des manuels de FLE approuvés par le Ministère de l'Éducation et utilisés en contexte scolaire prétendent être en conformité avec le Cadre européen commun de référence tout en affichant le niveau de progression linguistique sur leurs pages de couverture. Les manuels mettent aussi l'accent sur la maîtrise des compétences grammaticales de la part des apprenants mais souvent au détriment de la maîtrise des compétences de communication. Qui plus est, la grammaire est perçue comme un domaine peu créatif laissant très peu de place à l'expression personnelle de l'apprenant. En général, l'élève se montre beaucoup plus motivé pour le message qu'il reçoit que par les règles de grammaire qu'il doit mémoriser. Dans ce contexte, il s'avère que les besoins d'éducation supplémentaire en langue étrangère en dehors de l'enseignement formel ne concernent pas la grammaire mais le manque de communication, surtout orale, en langue étrangère.

Ayant pris tout cela en considération, nous nous sommes penchées dans cet article sur l'analyse de deux manuels de FLE qui sont utilisés dans l'enseignement primaire en Macédoine. Notre objectif était de vérifier si les contenus grammaticaux dans ces manuels correspondaient aux recommandations du CECRL, c'est-à-dire si la grammaire a une fonction communicative, quel type de grammaire y est présent et par quel type d'exercices le contenu grammatical est adopté. Nous sommes conscientes que l'approche du professeur joue également un grand rôle dans l'enseignement de la grammaire mais le manuel reste cependant son point de départ. 


\section{LA GRAMMAIRE DANS LE CECRL}

Ce n'est pas une nouveauté que les principes d'élaboration de programmes, d'examens et de manuels de FLE dans toute l'Europe sont définis par le Cadre européen commun de référence pour les langues (CECRL) destiné à tous les professionnels de langues vivantes dans l'objectif de les aider à surmonter les difficultés résultant de la diversité des systèmes éducatifs en Europe. Partant des composants du Cadre, on s'aperçoit qu'en effet il n'inclut pas de contenu grammatical se référant explicitement à un niveau linguistique particulier qu'on veut atteindre, mais définit un schéma descriptif des compétences linguistiques et communicationnelles permettant aux apprenants de réaliser des actions. En fait, la description du niveau de l'apprenant se fait en termes de plusieurs tâches qu'il doit accomplir correctement. Dans ce contexte, une continuité s'établit entre les tâches effectuées au sein du cours et celles que l'apprenant devrait accomplir par l'emploi de la langue dans la vie réelle. La perspective actionnelle prônée par le CECRL répond aux besoins de l'individu en tant qu'apprenant mais aussi et en tant qu'usager de la langue. Plus concrètement :

La compétence grammaticale est la capacité de comprendre et d'exprimer du sens en produisant et en reconnaissant des phrases bien formées selon ces principes et non de les mémoriser et de les reproduire comme des formules toutes faites. En ce sens, toute langue a une grammaire extrêmement complexe qui ne saurait, à ce jour, faire l'objet d'un traitement exhaustif et définitif (CECRL, 2001 : 89).

Dans cette perspective, la grammaire selon l'approche actionnelle devrait être perçue comme une compétence contribuant en premier lieu à l'accomplissement pratique d'une certaine tâche linguistique, qui sera ensuite appliquée dans une tâche sociale plus large dans laquelle l'étudiant serait un participant actif.

\section{GRAMMAIRE IMPLICITE ET GRAMMAIRE EXPLICITE}

La différence entre la grammaire implicite et la grammaire explicite est très bien expliquée par Coste et Galisson : 
La grammaire explicite est fondée sur l'explication des règles par le professeur, suivie d'applications conscientes par les élèves alors que la grammaire implicite vise à donner aux élèves la maîtrise d'un fonctionnement grammaticale (variations morpho-syntaxiques par exemple), mais ne recommande l'explication d'aucune règle et élimine le métalangage, ne s'appuie que sur une manipulation plus ou moins systématique d'énoncés et de formes (Coste, Galisson, $1976: 206,245$ ).

En complément de cette définition on pourrait ajouter que la grammaire implicite ne signifie pas l'absence de la grammaire, mais plutôt sa présence cachée, de telle manière que l'apprenant n'a pas le sentiment d'apprendre une langue (Al-Khatib, 2008 : 5). S'agissant de la grammaire explicite, suite à l'acquisition du métalangage grammatical de la part de l'apprenant, on procède vers son application consciente à travers des exercices. Plus précisément: "Il s'agit d'enseignement / d'acquisition de la description grammaticale de la langue à travers son modèle métalinguistique (en utilisant sa terminologie dans une version originale ou simplifiée) (...), et l'approche pourrait être déductive (...) ou inductive (...) » (Besse, Porquier, $2001: 80$ ).

Dans le cadre de l'approche déductive, le professeur explique d'abord la règle de grammaire, puis l'étudiant fait des exercices pour appliquer les règles. En revanche, l'approche inductive comprend un travail réflexif de la part de l'étudiant, à travers l'observation d'un corpus qui conduit à la formulation d'hypothèses qui doivent ensuite être confirmées (Puren, 2001 : $15)$.

Les opinions divergent quant à savoir s'il faut appliquer la grammaire explicite ou implicite à l'enseignement des langues. Notamment, certains didacticiens avancent que la pratique exclusive de la grammaire implicite n'est pas suffisante en soi et pourrait conduire à des erreurs chez l'étudiant si les règles qu'il adopte ne sont pas contrôlées par le professeur (Hocini, 2018 : 5). D'autres préconisent la pratique de la grammaire explicite estimant qu'elle ne permet pas la fossilisation des erreurs grammaticales, et ses avantages par rapport à la conceptualisation des règles contribuent largement à l'apprentissage d'une langue étrangère (Cuq, Gruca, 2009 : 387, dans Uwizeye, 2011 : 21). Selon d'autres, la manière explicite d'enseigner la grammaire serait utile pour déterminer les règles de grammaire, mais uniquement si l'étudiant a déjà acquis une connaissance implicite de la langue qu'il est en train d'apprendre. 


\section{CORPUS ET METHODOLOGIE}

Dans le cadre de cet article nous analysons la place de la grammaire et la manière dont les contenus grammaticaux sont présents dans les manuels de FLE, Vite 2 et Le Mag 1. Ces derniers sont approuvés par le Ministère de l'Éducation macédonien pour être utilisés dans l'enseignement primaire. Plus précisément, le choix du manuel Le Mag est fait en 2011 pour les classes de sixième, tandis que le manuel Vite est approuvé en 2012 (Vite 2) et en 2013 (Vite 3) pour les classes de septième et de huitième.

Dans le cadre de l'analyse que nous avons faite, nous nous sommes penchées sur plusieurs aspects liés à la manière dont la grammaire est présentée dans les manuels examinés. En premier lieu nous voulions savoir quelle approche est utilisée dans la présentation et l'adoption de la grammaire (implicite, explicite, inductive ou déductive), quels types d'exercices de grammaire y sont proposés et si la grammaire suit les recommandations générales du CECRL pour l'acquisition de compétences linguistiques par l'approche actionnelle, c'est-à-dire si les contenus grammaticaux sont présentés en fonction de la réalisation d'une tâche particulière.

\section{LA GRAMMAIRE DANS LE MANUEL VITE 2}

Le manuel Vite, édité par ELI en 2011, est une méthode de FLE pour adolescents qui propose une progression linguistique à quatre niveaux et qui se veut en conformité avec le CECRL. Cependant, on remarque tout de suite que le niveau n'est pas affiché sur la couverture comme c'est le cas avec la plupart des manuels de FLE. Ce n'est qu'indirectement, à travers le tableau des niveaux affiché au recto de la couverture, que l'on peut conclure que les manuels Vite 2 et Vite 3 sont destinés pour atteindre les niveaux A2 (Vite 2) et A2 / B1 (Vite 3). Si l'on prend en considération qu'un nouveau programme pour le FLE a été élaboré pour le niveau primaire selon lequel il est prévu d'atteindre le niveau $\mathrm{A} 1$ en classe de septième et le niveau $\mathrm{A} 2$ en huitième, il est à noter que le manuel Vite 2 ne correspond pas aux contenus prévus par le programme.

Dans le Guide pédagogique, les auteurs avancent que le manuel est élaboré en conformité avec les recommandations du CECRL et que les contenus suivent l'approche actionnelle selon laquelle l'apprenant n'est pas considéré uniquement comme un sujet au centre du processus d'apprentissage, mais comme un acteur social qui pourrait utiliser la langue étrangère pour 
accomplir des actions sociales, c'est-à-dire des tâches (Crimi, Blondel \& Hatuel, 2011b : 5).

L'espace consacré à la grammaire dans ce manuel est égal à l'espace dédié aux autres contenus linguistiques, soit une double page. La rubrique grammaticale, nommée La grammaire, c'est facile est précédée de rubriques destinées à l'acquisition du vocabulaire et à la maîtrise des compétences de communication. Il est également prévu d'effectuer divers exercices de systématisation des savoirs grammaticaux suite à chaque troisième leçon dans la section intitulée Je fais le point.

En dehors de la grammaire présente dans les leçons mêmes, à la fin du manuel on trouve un tableau de conjugaisons des verbes les plus courants. À part cela, on n'y trouve pas de contenu additionnel résumant les règles de grammaire.

On remarque également que dans la rubrique sur les compétences communicatives, les points de grammaire préalablement abordés sont souvent absents ou sont assez peu appliqués. À savoir, dans la première leçon, les pronoms objets directs, les formes de présentation et les adjectifs démonstratifs ne sont pas du tout présents dans la section écouter et lire, et dans les parties parler et écrire les instructions ne font pas référence à leur utilisation dans la production orale et écrite des élèves. Il en est de même pour la deuxième leçon où une attention insuffisante est accordée aux adverbes de quantité et au pronom adverbial en. Nous recommandons à l'enseignant de prêter plus d'attention à l'application des structures grammaticales précisément dans les compétences de communication parce que leur application concrète y est la plus évidente et parce que ces exercices simulent une situation linguistique réelle dans laquelle l'élève pourrait se trouver.

Selon les auteurs, les structures grammaticales contenues dans le manuel seraient « simples et rigoureuses, présentées de manière inductive et déductive afin de ne pas favoriser une voie d'adoption, au détriment d'une autre » (Crimi, Blondel \& Hatuel, 2011 : 5, 15). Ils précisent également que les tableaux expliquant les formes linguistiques et contenant les règles d'utilisation sont souvent précédés d'exemples tirés du dialogue d'introduction afin d'en assurer la contextualisation.

À notre avis, il serait beaucoup plus utile de prendre de tels exemples plus souvent lors de l'enseignement de la grammaire car de cette manière, reliant la structure et la règle, l'élève s'en souviendrait beaucoup plus facilement et en ferait l'usage dans un contexte similaire. D'après le tableau ci- 
dessous, on peut remarquer que seule la grammaire explicite est proposée dans le manuel et dans la plupart des leçons elle est enseignée de manière déductive.

Tableau 1. Démarche méthodologique dans le manuel Vite 2

\begin{tabular}{|c|c|c|c|c|}
\hline \multirow{2}{*}{$\begin{array}{l}\text { Manuel } \\
\text { VITE } 2\end{array}$} & \multicolumn{4}{|c|}{ Démarche méthodologique } \\
\hline & Inductive & Déductive & Implicite & Explicite \\
\hline Unité 1 & & + & & + \\
\hline Unité 2 & + & & & + \\
\hline Unité 3 & & + & & \\
\hline Unité 4 & + & + & & \\
\hline Unité 5 & & + & & \\
\hline Unité 6 & & + & & \\
\hline Unité 7 & & + & & \\
\hline Unité 8 & + & & & \\
\hline Unité 9 & & + & & \\
\hline
\end{tabular}

APPROCHE METHODOLOGIQUE DE L'ENSEIGNEMENT DE LA GRAMMAIRE DANS LE MANUEL VITE 2

Dans les cas où l'on propose une approche déductive explicite, la manière de procéder est traditionnelle avec des règles d'utilisation données immédiatement, sans phase destinée à la conceptualisation, suivies d'exercices d'application :

\section{Les pronoms relatifs qui et que}

- Les pronoms relatifs permettent de mettre en relation deux phrases.

- Le pronom qui, sujet, s'emploie pour les objets et pour les personnes et ne s'apostrophe jamais.

\section{Le garçon qui arrive est Marc}

- Le pronom que, complément d'objet direct, s'emploie pour les objets et pour les personnes.

\section{La jupe que je veux est la rouge.}

\section{Complète les phrases avec un pronom relatif.}

1. Voilà le livre je dois lire.

(Crimi et al. A, 2011a : 98) 
Dans le cadre des leçons où une approche inductive explicite est suggérée, avant que les règles ne soient énoncées l'étudiant effectue un exercice dans lequel une partie du dialogue introductif est reprise, mais il doit aussi en reprendre les éléments grammaticaux. De cette façon, l'étudiant se dote d'un aperçu de la règle grammaticale, mais même ici, la conceptualisation fait défaut. L'exercice est suivi d'une explication explicite des règles de grammaire adoptées et d'un exercice de réinvestissement :

\section{L'article partitif}

Relis le dialogue de la page 24 et complète les phrases

Pour faire de la mousse... (1) __ chocolat noir, (2) œufs, (3) beurre et (4) sucre, (5) crème fraîche.

\begin{tabular}{|l|l|l|}
\hline & Singulier & Pluriel \\
\hline Masculin & du/ de l' & \\
\cline { 1 - 2 } Féminin & de la/ de l' & des \\
\hline
\end{tabular}

L'article partitif exprime une quantité indéterminée et se forme avec la préposition de

+ les articles définis [...].

\section{Complète les phrases avec l'article partitif.}

1. Tu achètes du pain et œufs.

(Crimi et al. A, 2011a : 30)

Nous sommes d'avis que dans l'enseignement inductif, il faudrait introduire la phase de conceptualisation dans laquelle l'étudiant s'initierait à réfléchir de manière autonome et à découvrir la règle grammaticale qu'il devrait ensuite appliquer. Il pourra ainsi participer activement au processus d'apprentissage, comme indiqué dans le CECRL. Selon nous, dans les cas où des exemples sont repris au dialogue introductif, il est possible de procéder aussi d'une approche implicite. De cette manière, en suivant l'exemple donné, l'étudiant termine l'exercice donné sans être accablé de règles grammaticales, et l'enseignant n'emploie pas le métalangage grammatical. Ainsi, l'étudiant acquiert la grammaire de manière implicite et intériorisée sans se sentir obligé de mémoriser des règles.

S'agissant des exercices de grammaire, différents types sont recommandés par le CECRL : textes lacunaires, construction de phrases sur un modèle donné, choix multiples, exercices de substitution dans une catégorie, 
combinaison de phrases, traduction de phrases de L1 à L2, questions / réponses entrainant l'utilisation de certaines structures, exercices de développement de l'aisance langagière centrés sur la grammaire. La plupart de ces exercices sont contenus dans le manuel Vite 2 mais on note une absence totale d'exercices demandant une traduction du français vers la langue maternelle et une très faible présence d'exercices de développement de l'aisance langagière. Il est à noter aussi que les exemples contenus dans les exercices grammaticaux ne sont pas toujours thématiquement liés à la leçon introductive.

\section{LA GRAMMAIRE DANS LE MANUEL LE MAG 1}

Le manuel Le Mag 1, édité par Hachette en 2006, est une méthode de FLE s'adressant aux jeunes adolescents et proposant une démarche actionnelle où l'apprenant met en œuvre toutes ses compétences afin d'accomplir un ensemble de tâches. Le Mag 1 s'appuie sur les recommandations du CECRL et prépare l'apprenant au niveau A1 qui est affiché sur la couverture du manuel.

Les auteurs du manuels, Céline Himber, Charlotte Rastello et Fabienne Gallon, déclarent que le manuel est élaboré en conformité avec les recommandations du CECRL et que les contenus qui y sont présentés suivent l'approche actionnelle d'apprentissage des langues vivantes (Himber, Rastello \& Gallon, 2006a : 12).

Le manuel est composé de 9 unités didactiques partant de 0 jusqu'à 8 qui développent des compétences linguistiques, communicatives et culturelles chez les apprenants. Chaque unité didactique comprend 8 pages qui incluent les objectifs de l'apprentissage et les rubriques linguistiques. Le point de départ des unités est un document authentique qui présente une situation de communication authentique. La première double page du manuel est consacrée à la compréhension et à la production orale, suite à laquelle on note des savoirs lexicaux et grammaticaux qui sont abordés en contexte communicatif et qui visent à développer les compétences de compréhension et de production écrite chez les apprenants. Dans cette double page, la grammaire inductive est favorisée en forme de contenu qui résume les règles grammaticales de l'unité.

La rubrique grammaticale, nommée Atelier de langue, est censée favoriser la systématisation des savoirs grammaticaux et la maîtrise du vocabulaire appris à travers des exercices de réemploi. À la fin de l'unité 
didactique, on trouve une vraie mise-en-œuvre des compétences linguistiques des apprenants où ils effectuent des tâches langagières selon le modèle du niveau A1 de l'examen DELF.

En dehors de la grammaire présente dans les leçons mêmes, à la fin du manuel on trouve un tableau de conjugaisons des verbes les plus courants, y compris une liste des actes de parole abordés dans les unités didactiques de ce manuel.

D'après les auteurs, les structures grammaticales contenues dans ce manuel sont toujours présentées en situation contextualisée. Ils précisent que :

Le choix des différents points de grammaire tient compte de leur utilité dans la mise en œuvre d'une fonction de communication précise. Les dialogues supports dans la première double page de chaque unité permettent d'introduire les points de grammaire qui sont ensuite abordés en classe de manière inductive par le biais d'activités de recherche » (Himber-Rastello \& Gallon, $2006: 12$ ).

Selon nous, l'objectif n'est pas de mettre l'accent sur l'enseignement de la grammaire aux élèves, mais de leur permettre de réfléchir sur le fonctionnement des éléments linguistiques de la langue française.

Suite à cela, un exercice de conceptualisation est proposé, qui consiste en recherche des éléments linguistiques étudiés dans le texte de départ (dialogue ou document écrit authentique), formulation de la règle de la part des apprenants, et ensuite, fixation de la règle grâce à un tableau ou un récapitulatif pour en assurer une mémorisation rapide.

À notre avis, la grammaire est enseignée de manière inductive, reliant la structure du document authentique et la règle de grammaire, ainsi l'élève s'en souvient facilement et en fait l'usage dans un contexte de communication similaire. D'après le tableau ci-dessous, on peut remarquer que les auteurs ont prévu de permettre aux élèves la maîtrise d'un fonctionnement grammatical sans utiliser le métalangage ou l'utiliser de façon très limitée, mais qui va vers l'explication de la règle à la fin. Donc, dans ce manuel la grammaire implicite est proposée et elle est enseignée de manière inductive. 
Tableau 2 : Démarche méthodologique dans le manuel Le Mag 1

\begin{tabular}{|l|l|l|l|l|}
\hline \multirow{2}{*}{ Manuel } & \multicolumn{4}{|c|}{ Démarche méthodologique } \\
\cline { 2 - 5 } & Inductive & Déductive & Implicite & Explicite \\
\hline Unité 0 & + & & + & \\
\hline Unité 1 & + & & + & \\
\hline Unité 2 & + & & + & \\
\hline Unité 3 & + & & + & \\
\hline Unité 4 & + & & + & \\
\hline Unité 5 & + & & + & \\
\hline Unité 6 & + & & + & \\
\hline Unité 7 & + & & + & \\
\hline Unité 8 & + & & + & \\
\hline
\end{tabular}

APPROCHE MÉTHODOLOGIQUE DE L'ENSEIGNEMENT DE LA GRAMMAIRE DANS LE MANUEL LE MAG 1

L'objectif des auteurs de présenter les structures grammaticales de façon inductive se résume à l'initiation à une réflexion autonome et une découverte de la règle grammaticale qu'il devrait appliquer dans une situation de communication authentique. Ainsi, en suivant l'exemple donné, l'apprenant fait l'exercice et acquiert la grammaire de manière implicite et intériorisée sans se sentir obligé de mémoriser des règles. Si on prend l'exemple de la page 22 , on note un document écrit qui représente un reportage scolaire dans lequel la classe d'Émilie est décrite :

Émilie adore le sport. Elle a cours au collège sport - études de Modane dans la classe spécial-ski.

Qu'est-ce que c'est la classe spécial-ski ?

On a cours de maths, français, anglais, musique etc. Mais le mercredi et le vendredi, c'est spécial : on a trois heures de sport. Moi, j'ai cours de ski, c'est génial !

(Himber et al., 2006a : 22)

Observe le texte et complète avec le verbe avoir :

a) J'.........cours de ski.

b) On....... trois heures de sport.

(Himber et al., 2006a : 23)

Tous les points grammaticaux sont accompagnés d'une série d'exercices de réemploi et de systématisation des savoirs, présentés à la page 
Atelier langue qui privilégie la clarté de la règle afin de se concentrer sur l'aspect communicatif des savoirs acquis :

\section{Atelier langue \\ Le verbe "avoir"}

5. Complète avec le verbe avoir.

a) On....... Deux heures de sport.

b) Rémi ....... cours de maths le lundi.

(Himber et al., 2006a : 24)

Dans ce manuel, on note différents types d'exercices : textes lacunaires, construction de phrases sur un modèle donné, choix multiples, exercices de substitution dans une catégorie, combinaison de phrases, questions / réponses entraînant l'utilisation des structures grammaticales acquises etc. Tous ces exercices, pris en considération dans l'unité complète, visent à procéder à un apprentissage par tâches, comme le préconise le CECRL.

Nous sommes d'avis que les activités de grammaire proposées dans ce manuel mènent à l'accomplissement de tâches réelles et correspondent à la progression du niveau A1 du français. La grammaire est contextualisée, les exemples contenus dans les exercices grammaticaux sont toujours liés au thème de l'unité didactique et cela entraîne à une aisance langagière chez les apprenants.

\section{REMARQUES FINALES}

Suite à l'analyse comparée des manuels Vite 2 et Le Mag 1, utilisés dans l'enseignement primaire en Macédoine, on peut conclure que le Le Mag 1, qui n'est exploité qu'en classe de sixième, suit dans une bien plus grande mesure les préconisations du CECRL concernant l'introduction et l'acquisition de la grammaire. Plus précisément, les auteurs de ce manuel insistent sur l'objectif communicatif de la grammaire et sur son acquisition à travers la réalisation de tâches. Grâce à l'approche inductive préconisée, l'apprenant est y perçu comme un participant actif dans la découverte des règles grammaticales et dans leur conceptualisation devenant ainsi beaucoup plus conscient de l'application pratique de ces règles dans des situations linguistiques concrètes. Par contre, le manuel Vite 2, utilisé en classes de septième et de huitième, présente les contenus grammaticaux d'une manière explicite et déductive, ne permettant 
pas à l'élève d'être suffisamment induit pour découvrir les règles de manière autonome. En outre, les règles de grammaire ne sont pas suffisamment réinvesties dans la partie destinée au développement des compétences langagières où leur application concrète devrait être la plus accentuée. Quant aux règles de grammaire, on a pu conclure que les deux manuels disposent d'une offre variée en conformité avec les recommandations du CECRL.

Même si le CECRL ne donne pas d'orientations concrètes sur l'enseignement des contenus grammaticaux, laissant ainsi la possibilité d'appliquer et de combiner diverses approches méthodologues, il préconise cependant que la grammaire ne soit une fin en soi et qu'elle soit dotée d'une fonction communicative.

Nous sommes d'avis que la meilleure façon d'y arriver c'est de pratiquer un enseignement combiné : explicite inductif ou implicite inductif. Il est clair que l'enseignant peut choisir l'approche qu'il considère la plus appropriée quelle que soit la manière dont la grammaire est présentée dans le manuel, mais de toute manière c'est le manuel qui reste son point de départ. L'analyse montre que le marché offre des manuels de FLE de qualité, en conformité aux recommandations modernes sur l'adoption de la grammaire française. Sachant qu'en Macédoine la procédure de sélection de nouveaux manuels pour le primaire est en cours, nous espérons que cette analyse servira d'orientation pour en choisir les plus appropriés.

Milena Kasaposka-Chandlovska, Maja Milevska-Kulevska

THE GRAMMAR IN THE FRENCH AS FOREIGN LANGUAGE COURSE BOOKS USED IN THE PRIMARY EDUCATION IN MACEDONIA

\section{Summary}

Taking into consideration that the number of students learning French in the primary education in Macedonia continued falling in the past years and the fact that this reduced interest has direct implications on learning French in the upper education levels, we need to analyze the reasons for this situation. When asked about the reasons for not choosing French in the primary education process, and especially at university level, the students provided the complex French grammar as the most common reason. We believe that the very fact that the first thing the students associate with the French language is its grammar, points that apart from the contemporary methodological approaches recommended by the Common European Framework of Reference for Languages since 2001, there is a high probability that the grammar in the French 
language course books does not have a communicative goal, but is instead taught in the traditional manner. This situation urged us to analyze the grammatical context in two French language text books, Le Mag and Vite which are used in the Macedonian primary education. Our analysis will primarily provide a broader picture of how the grammar is presented in those two course books and will inquire to what extent it corresponds with the CEFRL recommendations for development of communicative competences. Furthermore, we will analyze the grammatical approach present in the course books and grammar exercises proposed for its adoption.

Key words: grammar, course books, French, primary education, Macedonia.

\section{RÉFÉRENCES BIBLIOGRAPHIQUES}

Al-Khatib, M. (2008). Faut-il ignorer la grammaire explicite? Colloque international : Enseigner les structures langagières en FLE. Bruxelles : Université Libre de Bruxelles. Disponible sur : https://grammfle.ulb.ac.be/fichiers/colloques/Nantes2008/ALKHATIB.pdf

Besse, H.-Porquier, R. (2001). Grammaires et didactiques des langues. Paris : Hatier-Crédif, 1984, 86.

Cadre européen commun de référence pour les langues : apprendre, enseigner, évaluer. (2001). Paris: Conseil de l'Europe / Les Éditions Didier. Disponible sur : https://rm.coe.int/16802fc3a8

Coste, D.-Galisson, R. (1976). Dictionnaire de didactique des langues, Paris : Hachette.

Fougerouse, M.-Ch. (2001). L'enseignement de la grammaire en classe de français langue étrangère. Études de linguistique appliquée, 122, 165178. Doi : https://doi.org/10.3917/ela.122.0165

Günday, R.-Cakir, F. \& Atmaca, H. (20. déc. 2017). Quelle démarche suivre en didactique de langue étrangère : La grammaire implicite ou la grammaire explicite ? Humanitas, volume 5, issue 10, p.247-262. DOI :10.20304/humanitas.292394

Hocini, Z. (2018). L'enseignement / apprentissage de la grammaire dans le collège algérien : quelle place pour la démarche inductive ? Multilinguales, 9.9 Disponible sur: https://journals.openedition.org/multilinguales/1417

Puren, C. (2001). Outils et méthodes d'analyse des manuels de langue. L'exemple des procédures d'enseignement / apprentissage de la grammaire.

http://s9577412bcd03c8a2.jimcontent.com/download/version/0/mo 
dule/4304418351/name/PUREN_2001h_Outils_analyse_grammaire_da ns_manuels.pdf

Trajkova, M.-Velevska, M. (2017). La place et l'évaluation de la grammaire dans les méthodes destinées à l'enseignement / apprentissage du FLE. In : Nikodinovska, R. (éd.) (2017). Assessement in Foreign Language and Literature Teaching. Skopje: Filološki fakultet „Blaže Koneski“. 519-533. Disponible sur : https://www.researchgate.net/publication/323228058_Assessment_in _foreign_language_and_literature_teaching_Evaluacija_vo_nastavata_po_ stranski_jazici_i_knizevnosti_ed_Radica_Nikodinovska_Filoloski_fakulte t_Blaze_Koneski_Skopje_2017_571_p

Uwizeye, M-L. (2011). Les exercices de grammaire et le développement de la compétence communicative : une typologie. Varen : Universitetet i Oslo. Disponible sur : https://www.duo.uio.no/handle/10852/25687

Vincent, F.-Dezutter, O. \& Pascale, L. (2013). Enseigner la grammaire selon une approche inductive ou déductive ? Québec français, 170, 93-94. Disponible sur : https://www.researchgate.net/publication/284031822_Enseigner_la_ grammaire_selon_une_approche_inductive_ou_deductive

\section{MANUELS :}

Crimi, A.-M.-Blondel M. \& Hatuel D. (2011a). Vite 2. Paris : ELI.

Crimi, A.-M.-Blondel M. \& Hatuel D. (2011b). Vite 2 : Guide pédagogique. Paris : ELI

Himber, C.-Rastello, C. \& Gallon, F. (2006a). Le Mag 1. Paris : Hachette.

Himber, C.-Rastello, C. \& Gallon, F. (2006b). Le Mag 1 : Guide pédagogique. Paris : Hachette. 
